\title{
铝合金大功率超声波焊接界面及接头性能研究
}

\author{
李 欢 ${ }^{1,2}$ 周 亢 $^{3}$ 曹 虎 $^{2}$ 张锦洲 $^{1}$ \\ (1. 长江大学机械工程学院 荆州 434023; \\ 2. 华南理工大学机械与汽车工程学院 广州 510640; \\ 3. 北京理工大学机电学院 北京 100081)
}

\begin{abstract}
摘要: 尽管大功率超声波焊接更适合异种金属的焊接, 在汽车轻量化上应用的研究较少。研究 6061-T6 铝合金与纯铜、6061-T6 铝合金与 DC04 钢大功率超声波焊接的界面及接头性能, 主要包括界面的温升、中间相的生成以及接头的力学性能、拉伸断 口形貌。结果表明, 在钢/铝超声波焊接中, 在焊接时间 $1 \mathrm{~s}$ 、压力 $1.98 \mathrm{kN}$ 和振幅 $27 \mu \mathrm{m}$ 时, 界面生成厚度为 $1.70 \mu \mathrm{m}$ 、主要 成分为 $\mathrm{FeAl}_{3}$ 和 $\mathrm{Fe}_{2} \mathrm{Al}_{5}$ 且连续分布的中间相; 近一半的超声功率转化为界面能量; 焊接区域面积明显大于焊头端面; 界面发 生了较大的塑性变形, 存在明显的机械互锁; 接头的最高强度为 $3.95 \mathrm{kN}$, 拉伸破坏行为为界面式破坏, 且断裂模式为㓞性脆性复合断裂。在铜/铝焊接中, 在焊接时间 $0.54 \mathrm{~s}$ 、压力 $1.45 \mathrm{kN}$ 和振幅 $25 \mu \mathrm{m}$ 时, 界面生成厚度为 $1.95 \mu \mathrm{m}$ 、主要成分为 $\mathrm{Al}_{2} \mathrm{Cu}$ 的中间相, 获得的接头最高强度为 $3.20 \mathrm{kN}$, 其拉伸破坏行为为拉拔式破坏。研究了中间相厚度与接头强度的关系, 适 当的 IMC 厚度能促进连接。
\end{abstract}

关键词: 铝合金; 超声波焊接; 力学性能; 断口形貌; 中间相

中图分类号: TG453

\section{Analysis of Welding Interface and Joint Properties of High Power Ultrasonic Welding of Aluminum Alloy}

\section{Huan $^{1,2}$ ZHOU Kang ${ }^{3}$ CAO Biao ${ }^{2}$ ZHANG Jinzhou ${ }^{1}$}

(1. School of Mechanical Engineering, Yangtze University, Jingzhou 434023;

2. School of Mechanical and Automotive Engineering,

South China University of Technology, Guangzhou 510640;

3. School of Mechatronical Engineering, Beijing Institute of Technology, Beijing 100081)

\begin{abstract}
Although high-power ultrasonic welding(USW) is better suitable for joining dissimilar metals, there is few studies on application of this welding method in automobile lightweight. The interface performances and joint properties, including interface temperature rise, intermetallic compound (IMC) growth, joint strength and fracture morphology, in 6061-T6 aluminum alloy dissimilar high-power USW are studied. In USW of Steel/Al combination, at $1 \mathrm{~s}$ welding time, $1.98 \mathrm{kN}$ normal force, and $27 \mu \mathrm{m}$ amplitude, a continuous IMC layer with a thickness of $1.7 \mu \mathrm{m}$ occurs at welding interface. The IMC is primarily composed of $\mathrm{FeAl}_{3}$ and $\mathrm{Fe}_{2} \mathrm{Al}_{5}$. About half of the ultrasonic power is converted into interfacial energy. The welding zone is significantly larger than the sonotrode tip area. High plastic deformation and obvious mechanical interlocking occur at the interface. The obtained highest lap-shear strength of Steel/Al joint were $3.95 \mathrm{kN}$. The tensile failure behavior of the joint is interface failure, and the fracture mode of the joint is ductile-brittle hybrid fracture. In USW of $\mathrm{Cu} / \mathrm{Al}$ combination, at $0.54 \mathrm{~s}$ time, $1.45 \mathrm{kN}$ normal force, and $25 \mu \mathrm{m}$ amplitude, the IMC layer with thickness of $1.95 \mu \mathrm{m}$ at the interface is primarily composed of $\mathrm{Al}_{2} \mathrm{Cu}$; the obtained highest lap-shear strength of $\mathrm{Cu} / \mathrm{Al}$ joint is $3.20 \mathrm{kN}$, and the tensile failure behavior of the joint is pull-out failure. The relationship between IMC thickness and joint strength shows that proper IMC layer thickness can improve the joints strength.
\end{abstract}

Key words: aluminum alloy; ultrasonic welding; mechanical properties; fracture morphology; intermetallic compound

* 国家自然科学基金 $(51175184,51605103)$ 和长江大学青年博士科研 (802100270402)资助项目。20200415 收到初稿, 20201101 收到修改稿 


\section{0 前言}

全球环境污染的形势日益严峻, 减轻汽车重量 是减少污染物排放的有效途径, 近年来汽车轻量化 已成为研究热点 ${ }^{[1]}$ 。车身减重和电缆系统减重是汽车 轻量化的两种有效手段 ${ }^{[2-3]}$ 。相比铜和钢, 铝合金具 有较低的密度以及良好的导电性, 因此在电缆和车身 上分别可以替代铜和钢。然而由于铝与铜以及铝与钢 之间的物理性能相差大, 导致铜/铝及钢/铝接头的焊 接性较差, 这给汽车轻量化带来了挑战。采用弧焊以 及激光焊在焊接铝合金异质金属时会生成较厚的脆 性中间相 ${ }^{[4]}$ 。采用电阻点焊焊接铝/铜比较困难, 而焊 接钢/铝时需要较大的能量输入且电极容易磨损 ${ }^{[}$。最 近铝合金搅拌摩擦点焊的研究较多 ${ }^{[6]}$, 但它过程较长 且在所需的顶锻力较大, 工具头容易磨损 ${ }^{[7]}$ 。

超声波焊接是在压力作用下将焊头的机械振动 能传递至界面使其塑性变形并发生冶金反应的一种 固相焊接方法, 它是一种绿色节能、环境友好的焊 接技术 ${ }^{[8]}$ 。在焊接过程没有烟尘和电弧产生, 不需 要添加焊剂, 焊接所需的能量一般低于电阻点焊的 $5 \%$ 以及搅拌摩擦点焊的 $25 \%{ }^{[9]}$; 同时, 超声软化降 低了工件塑性变形所需的力, 焊接压力一般低于 $2 \mathrm{kN}$, 能有利于控制工件的塑性变形并且减少工具 头的磨损; 此外, 超声波焊接属于固相焊接且过程 较短, 使得焊接微观组织变化小。上述优点使得超 声波焊接更适用于异质金属接头的连接。

但超声波焊接过程较短, 超声软化作用及工件之 间的高频摩擦使得界面反应复杂, 即使国内外学者开 展了众多对超声波金属焊接界面的研究, 目前对超声 波焊接机理认识也不足。此外, 钢/铝超声波焊接研 究也较少, 且由于钢硬度较高, 需使用大功率 $(2.5 \mathrm{~kW}$ 或以上)超声波焊机确保界面足够的塑性变形, 但目 前很少有 $4.0 \mathrm{~kW}$ 或以上功率的超声波焊机来进行钢/ 铝焊接的研究。本文研究了 6061-T6 铝合金异质接头 的大功率超声波焊接, 明晰界面行为包括焊接界面的 微观组织、断裂特征并获得较高的焊接强度。

\section{1 试验方法}

焊接试验采用 Telsonic M5000 型大功率超声波 金属焊机(图 1a), 其最大功率为 $4000 \mathrm{~W}$, 振动频率 为 $20 \mathrm{kHz}$ 。考虑到焊头容易粘铝, 且 6061-T6 铝合 金的屈服强度均高于纯铜和 DC04 钢, 将铝合金移 作下工件; 上工件分别为 T2 纯铜及 DC04 低碳钢。 所有工件被剪成 $100 \mathrm{~mm} \times 25 \mathrm{~mm}$ 的尺寸, 铜/铝焊接
时的工件厚度均为 $0.8 \mathrm{~mm}$; DC04 钢/6061-T6 铝合金 焊接时钢和铝厚度分别为 $0.8 \mathrm{~mm}$ 和 $1.2 \mathrm{~mm}$ 。工件之 间采用搭接方式放置, 搭接面积为 $25 \mathrm{~mm} \times 25 \mathrm{~mm}$ 。 在工具头底端带有两种不同齿数的焊头, 分别为 9 个梯形齿和 10 个梯形齿, 所有焊头端面面积均为 $7 \mathrm{~mm} \times 5 \mathrm{~mm}$, 如图 $1 \mathrm{~b} 、 1 \mathrm{c}$ 所示。

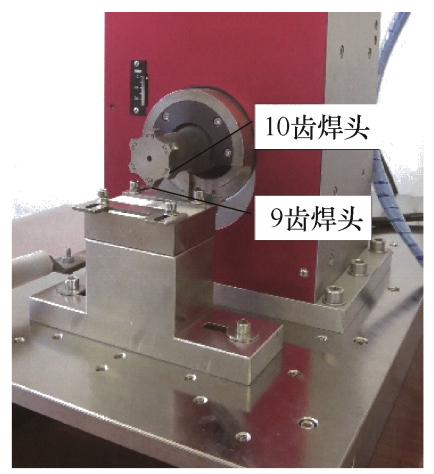

(a) 焊机

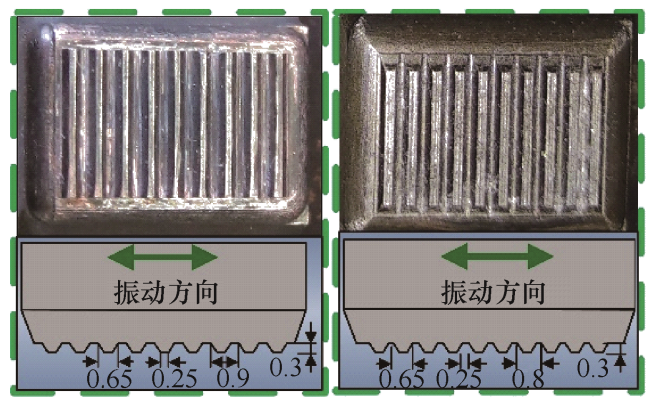

(b) 9齿焊头

(c) 10齿焊头

图 1 超声波焊接设备

试验中，对于钢/铝超声波焊接选用带 10 个齿 的焊头, 以避免刚度大的钢板被焊头压溃进而严重 破坏接头强度; 铜/铝焊接时选用 9 个齿的焊头。对 于铜/铝焊接, 工艺参数为振幅 $25 \mu \mathrm{m}$ 、焊接时间 0 $0.7 \mathrm{~s}$ 、焊接压力 $1.45 \mathrm{kN}$ 和焊头齿数 9 个; 对于钢/ 铝焊接, 工艺参数为振幅 $27 \mu \mathrm{m}$ 、焊接时间 $0 \sim 1.2 \mathrm{~s}$ 、 焊接压力 $1.98 \mathrm{kN}$ 和焊头齿数 10 个。焊接工艺参数 如表 1 所示。尽管振幅比例均设置为 $100 \%$, 在钢为 上工件时的焊头振幅要比铜高, 这是因为刚度大的 上工件(钢)更有利于焊头的振动 ${ }^{[10]}$ 。

表 1 超声波焊接工艺参数

\begin{tabular}{ccccc}
\hline & 振幅 $\zeta / \mu \mathrm{m}$ & 时间 $t / \mathrm{s}$ & 压力 $F_{\mathrm{N}} / \mathrm{kN}$ & 焊头齿数 $n$ \\
\hline 铜/铝 & 25 & $0 \sim 0.7$ & 1.45 & 9 \\
钢/铝 & 27 & $0 \sim 1.2$ & 1.98 & 10 \\
\hline
\end{tabular}

采用 $\mathrm{K}$ 型热电偶测量了界面温度, 为保证测量 精度, 选用直径 $0.1 \mathrm{~mm}$ 热电偶丝。测温点距离焊 接区域中心 $1.5 \mathrm{~mm}$, 测温方法如下: 在铝板的上 表面开 $0.5 \mathrm{~mm}$ 半圆形孔, 将热电偶塞入孔内, 然 后将热电偶丝嵌入材料内部, 图 2 为热电偶测温 示意图。 


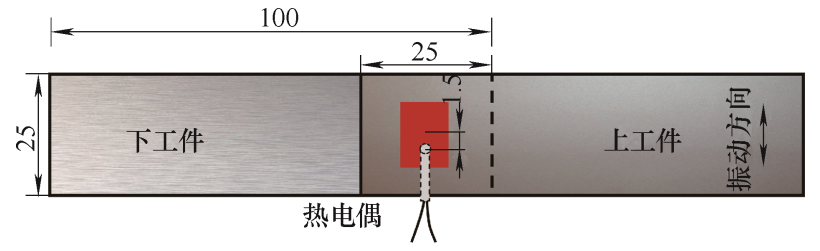

图 2 测温示意图 $(\mathrm{mm})$

采用装配有能量色散 $\mathrm{X}$ 射线光谱仪(Energy dispersive spectrometer, EDS)的扫描电子显微镜 (Scanning electron microscope, SEM) 观测了界面及 其拉伸断口的微观形貌。并进行了 $\mathrm{X}$ 射线衍射 (X-ray diffraction, XRD) 分析来进一步明确中间相成 分。XRD 光谱测量范围为 $20^{\circ} \sim 90^{\circ}$, 步长为 $0.2^{\circ}$ ，扫描速度为 $0.02^{\circ} / \mathrm{s}$ 。

由于超声波金属焊接区域极不规则, 很难计算焊接 区域面积, 拉伸剪切力是评价超声波焊接质量的主要手 段 ${ }^{[12]}$ 。对两种接头的超声焊接试样进行了拉伸剪切力测 试, 拉伸机为岛津 AGS-X 型微机控制电子万能试验机。 设定的拉伸速度为 $2 \mathrm{~mm} / \mathrm{min}$, 这能避免接头强度的破 坏和界面组织的变化 ${ }^{[11]}$ 。对于每一组工艺参数, 取三次 测试的平均值作为该参数下的拉伸剪切力。

\section{2 结果与讨论}

\section{1 界面温度}

界面温度揭示了冶金反应规律, 是焊接微观组 织演变最直接的证据 ${ }^{[13]}$ 。图 3 是界面峰值温度随焊 接时间的变化关系。随着焊接时间的增加, 铜/铝界 面温度在 $0.3 \mathrm{~s}$ 之后升至 $330{ }^{\circ} \mathrm{C}$, 之后逐渐升高, 并在焊接时间 $0.54 \mathrm{~s}$ 时达到峰值 $442{ }^{\circ} \mathrm{C}$ 。根据我们 之前的研究 ${ }^{[14]}$, 焊接区域中心温度会比热电偶测温 点高 $17{ }^{\circ} \mathrm{C}$, 这说明界面温度会达到 $451{ }^{\circ} \mathrm{C}$, 这温 度仅为 6061 铝合金熔点 $\left(650{ }^{\circ} \mathrm{C}\right)$ 的 $70 \%$, 且没超过 $\mathrm{Al}-\mathrm{Cu}$ 共晶转变的临界温度 $\left(546{ }^{\circ} \mathrm{C}\right)$ 。

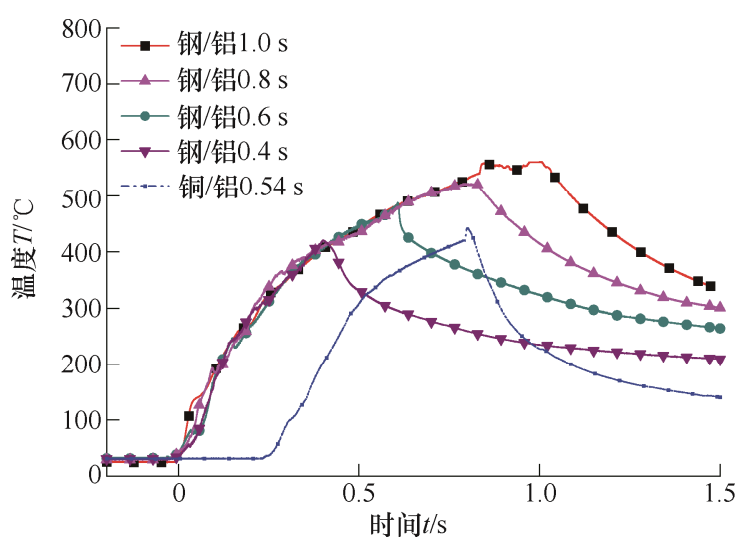

图 3 界面温度随时间变化

对于钢/铝焊接, 随着焊接时间的增加, 测量的
温度在时间 $0.4 \mathrm{~s} 、 0.6 \mathrm{~s}$ 和 $0.8 \mathrm{~s}$ 分别为 $420{ }^{\circ} \mathrm{C}$ 、 $487{ }^{\circ} \mathrm{C} 、 530{ }^{\circ} \mathrm{C}$, 并在 $1 \mathrm{~s}$ 时候温度达到 $560{ }^{\circ} \mathrm{C}$, 该 温度远高于常规低功率超声波焊接时的温度 ${ }^{[2,15]}$, 与 HADDADI 等 ${ }^{[16]}$ 报道的大功率超声波焊时温度相 近。焊接结束后, 钢/铝的冷却速度明显低于铜/铝焊 接时, 这是因为钢导热率远低于铜。

由于热电偶测温方法不能直接测量焊接区域中 心的温度, 且有关钢/铝超声波焊接过程模拟的研究 较少, 为了揭示界面的微观组织演变机理, 模拟了 焊接温度场分布。超声波金属焊接模拟中热源加载 方式有两种 ${ }^{[17]}$ : (1) 热源以摩擦热和塑性变形热方 式加载; (2) 以超声功率方式加载。模拟中的热源与 超声功率有关, 超声波焊接的输入能量 $E$ 由超声功 率 $P$ 决定, 其计算公式为

$$
E_{t}=\int_{0}^{t} \eta \times P(t) \times t \mathrm{~d} t
$$

式中, $t$ 为焊接时间 $P(t)$ 为电功率曲线, $\eta$ 为电功率 转变为热量的比例。加载至接触面的热流密度 $Q_{t}$ 计算公式为

$$
Q_{t}=\frac{\eta \times P(t)}{2 \times A_{w}}
$$

式中, $A_{w}$ 为接触区域面积, 模型中将接触区域近似 为矩形, 其面积根据拉伸断口(图 17a)实测得到。

图 4 为系统采集的超声电功率曲线, 曲线基本 平滑, 数据没出现明显的三角齿状波动, 这说明工 件表面粗鋉度分布较均匀。此外, 在焊接初期, 电 功率快速增加, 之后缓慢增加然后降低, 这也对应 了界面峰值温度的变化规律。尽管在时间 $0.54 \mathrm{~s}$ 内 钢/铝焊接时超声电功率要低于铜/铝焊接同时刻的 值, 但由于钢的导热率远低于铜, 导致在 $0.4 \mathrm{~s}$ 时热 电偶测得的钢/铝焊接温度与长时间 $(0.54 \mathrm{~s})$ 铜/铝焊 接时的温度近似。



图 4 电功率曲线

图 5 为建立的有限元模型。将 $Q_{t}$ 加载至工件接 
触区域。其他边界条件为: 对称面为绝热边界, 模 型中与空气所有接触面都定义为对流换热面; 由于 焊头及工件的高速振动, 产生了较大的空气对流换 热系数。焊头和工件对流换热系数分别设定为 $120 \mathrm{~J} /\left(\mathrm{m}^{2} \cdot{ }^{\circ} \mathrm{C}\right)$ 和 $500 \mathrm{~J} /\left(\mathrm{m}^{2} \cdot{ }^{\circ} \mathrm{C}\right){ }^{[14]}$, 在焊接结束时均为 $5 \mathrm{~J} /\left(\mathrm{m}^{2} \cdot{ }^{\circ} \mathrm{C}\right)$ 。假定室内温度稳定在 $25{ }^{\circ} \mathrm{C}$ 。为减少 模型计算量的同时保证精度, 模拟中设定电功率 转变为热量的比例 $\eta$ 值在 $0.20 \sim 0.80$ 且保留两位 小数, 并设定所有间隔 $0.1 \mathrm{~s}$ 的模拟的温度值与 热电偶测温值之间相对误差的平均值低于 $5 \%$ 时 停止计算。

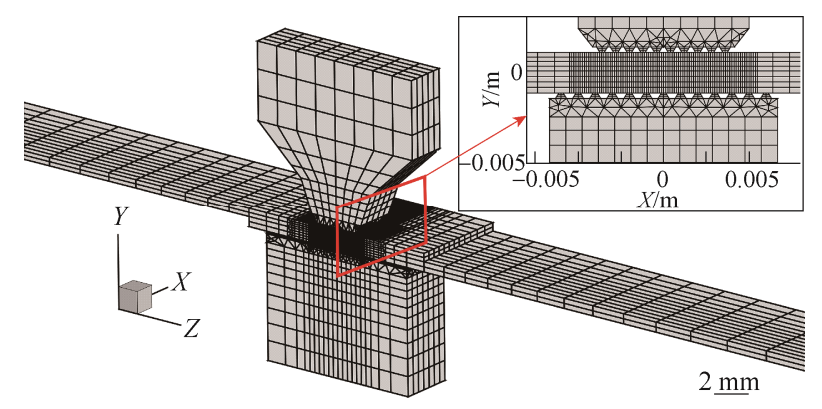

图 5 有限元模型

当超声功率与热量之间的转换系数 $\eta=0.51$ 时, 模拟温度结果与试验结果之间吻合较好, 相对误差 为 $4.972 \%$, 如图 6 所示。在该条件下模拟的在焊接 时间 $1 \mathrm{~s}$ 时的温度场分布结果如图 7 所示。此时最 高温度为 $556{ }^{\circ} \mathrm{C}$, 出现在工件接触面中心处。该温 度值低于铝合金的熔点 (约 $680{ }^{\circ} \mathrm{C}$ )。模拟的热电偶 测温点温度为 $547{ }^{\circ} \mathrm{C}$, 比焊接区域中心温度低 $9{ }^{\circ} \mathrm{C}$ 。由于钢、铝导热系数的差异, 工件之间温度 场呈现非对称分布, 且铝的等温线范围比钢宽, 但 在焊接区域范围内, 铜/铝接触面两端附近温度相 同。相比工件而言, 焊头上高温区域小。焊头上的 高温会阻碍超声波在金属中的传递, 但高温区域范 围窄，对超声的传播影响较小。

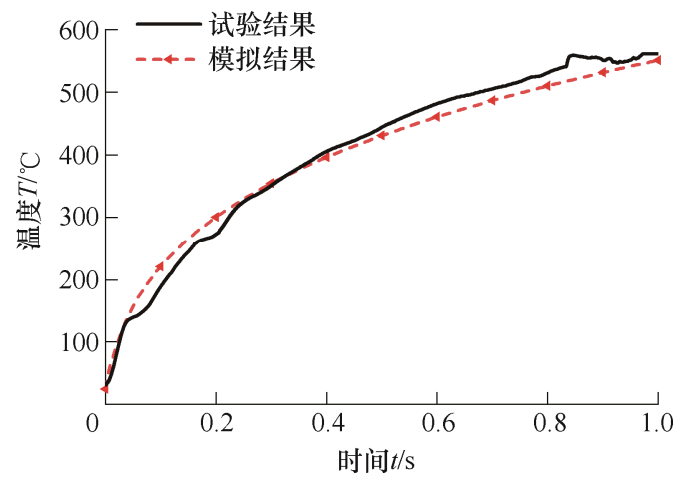

图 6 模拟温度结果与试验结果对比

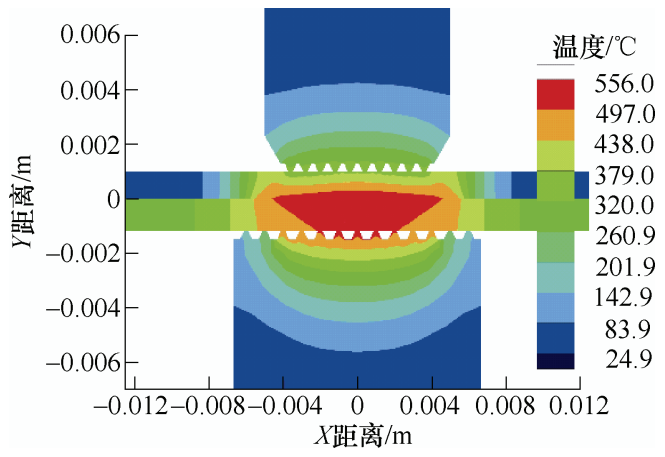

图 7 焊接横截面温度场分布

掌握温度场分布规律是研究界面扩散行为的前 提。图 8 为时间 $1 \mathrm{~s}$ 时的连接界面焊接区域温度分 布。焊接区域(Welding zone, WZ)沿 $X$ 向边角处的温 度为 $501{ }^{\circ} \mathrm{C}$, 低于界面中心处 $55{ }^{\circ} \mathrm{C}$ 。沿 $Z$ 向边缘 处低于界面中心 $57{ }^{\circ} \mathrm{C} 。 \mathrm{DC} 04$ 钢的动态再结晶温度 高于 $600{ }^{\circ} \mathrm{C}^{[18]}$, 这远高于材料的最高温度, 因此 在钢侧不会产生明显的热影响区(Heat affected zone, $\mathrm{HAZ}$ ); 铝的动态再结晶温度约为 $250{ }^{\circ} \mathrm{C}$, 根据界 面温度分布结果所预测的铝侧热影响区宽度为 $1.8 \mathrm{~mm}$ 。

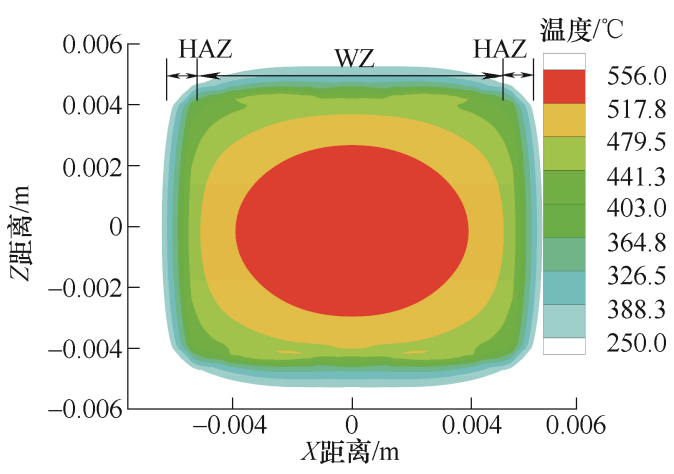

图 8 界面温度场分布

\section{2 铜/铝焊接微观组织与力学性能}

\subsection{1 焊接微观组织}

在图 9a 中展示了焊接时间 $0.54 \mathrm{~s}$ 时铜/铝超声 波焊接接头横截面的形貌。铜和铝侧均发生了嵌入。 在铜的一面, 焊头上的齿完全嵌入进了铜板表面导 致其厚度减小, 边缘处出现明显的母材挤出现象, 而底座齿并未完全嵌入铝表面。从图 $9 \mathrm{~b}$ 中可见在接 合处有明显的高度约 $50 \mu \mathrm{m}$ 的漩涡状塑性变形区, 但几乎没有发现任何空隙或裂缝, 这说明焊接质量 较高 ${ }^{[19]}$ 。图 $9 \mathrm{c}$ 是焊接时间 $0.54 \mathrm{~s}$ 形成的中间相形貌, 其厚度为 $1.95 \mu \mathrm{m}$ 。

为了明确界面中间相成分，对铜/铝接头断口的 $\mathrm{Cu}$ 侧进行了 XRD 分析, 如图 10 所示。结果表明, 在焊接中形成的中间相主要由 $\mathrm{Al}_{2} \mathrm{Cu}$ 组成, 这说明 并未出现中间相分层, 预示高的焊接接头强度。此 
外, 检测到部分铝残留在铜中, 这是因为铜/铝接头 强度明显高于母材铝的剪切强度, 部分铝被撕裂了 残留在 $\mathrm{Cu}$ 侧。在 $\mathrm{Al}$ 侧粘附的 $\mathrm{Cu}$ 越多, 形成的有 效微观接头越多, 接头强度也就越大。

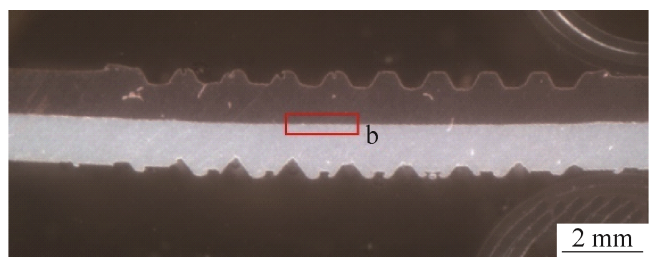

(a) 接头横截面形貌

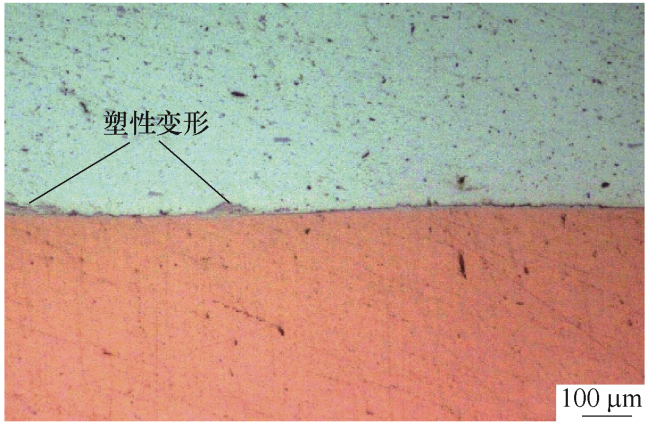

(b) 界面宏观形貌

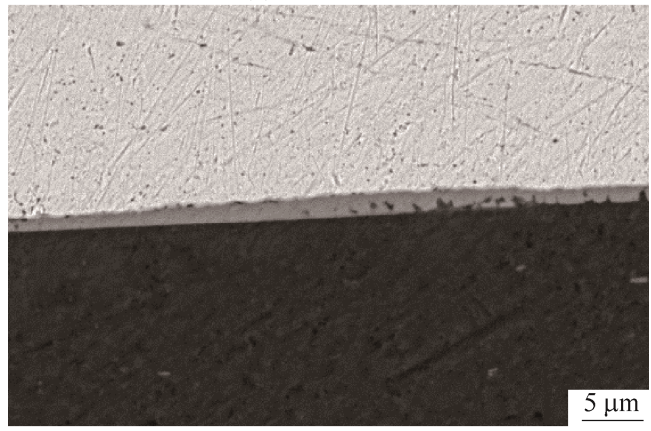

(c) 界面微观形貌

图 9 铜/铝焊接形貌

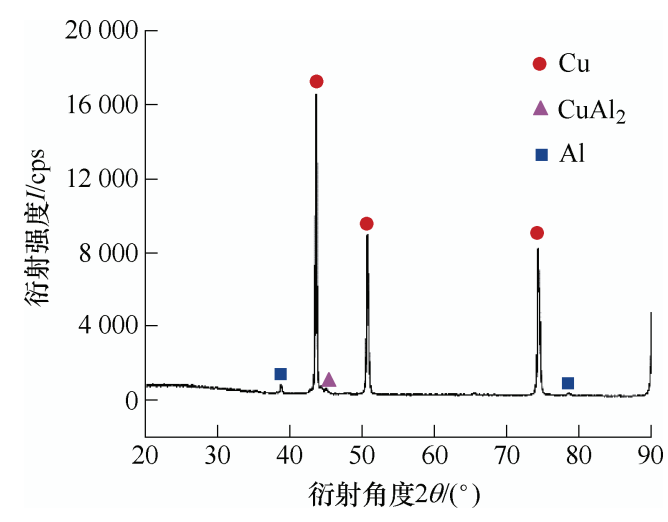

图 10 铜/铝接头断口 $\mathrm{Cu}$ 侧 XRD 分析结果

\subsection{2 断口形貌}

图 11 为 SEM 显微观测的不同焊接时间的铜/ 铝接头在 $\mathrm{Al}$ 侧的断裂形态图。断口区域由摩擦区域 和塑性变形区域组成。此外，随着焊接时间的增加， 焊缝的失效行为从焊接初始阶段的界面分离(图 11a) 转变为在 $0.54 \mathrm{~s}$ 时的熔核拔出(拉拔, Pullout) 模式(图
$11 \mathrm{~b})$, 接着在 $0.7 \mathrm{~s}$ 时又转变为界面分离(图 11c)。 铜/铝接头焊接面积与焊头端面近似。在断口处存在 大量韧窝(图 11d)同时也存在脆性断裂的特征。

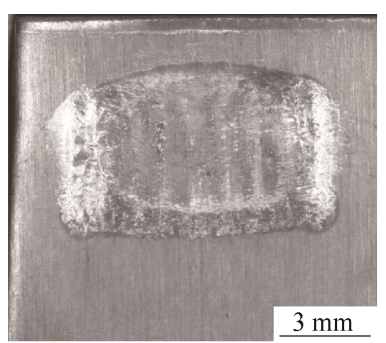

(a) $0.2 \mathrm{~s}$

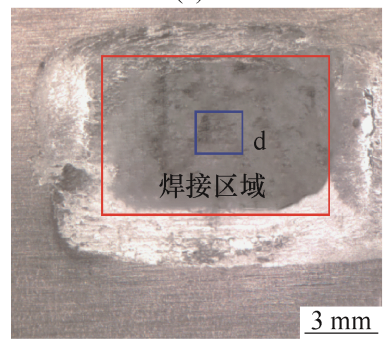

(c) $0.7 \mathrm{~s}$

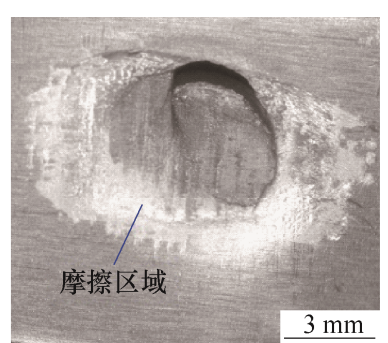

(b) $0.54 \mathrm{~s}$

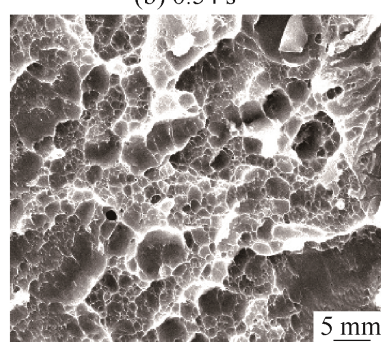

(d) 断口微观形貌
图 11 不同焊接时间的接头断口形貌

\subsection{3 接头力学性能}

图 12 是焊接接头的拉伸剪切强度与焊接时间 的关系。在 $0.54 \mathrm{~s}$ 时铜/铝焊接接头的最大拉伸剪切 强度达到约 $3.2 \mathrm{kN}$, 该强度达到了采用同样焊接设 备获得的 $\mathrm{Cu} / \mathrm{Cu}$ 的接头性能的 $75 \%$ 。高质量焊接 产生的原因是界面的 IMC 厚度在近似 $2 \mu \mathrm{m}$ 时会促 进冶金结合且不会降低焊接强度。

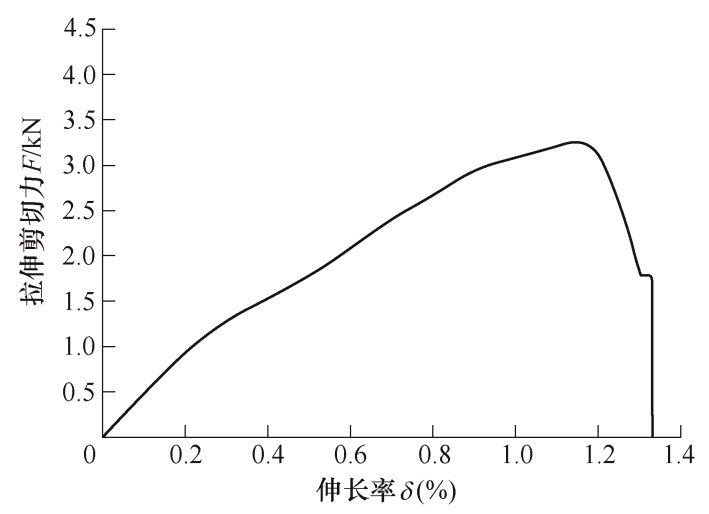

图 12 铜/铝接头拉伸曲线

\section{3 钢/铝焊接微观组织与力学性能}

\subsection{1 焊接微观组织}

对于钢/铝超声波焊接，同样由于焊头、底座 上的齿完全嵌入工件表面导致其厚度减小(图 13a)。 图 $13 b$ 是焊接时间 $1 \mathrm{~s}$ 时界面微观形貌, 由于钢/铝 界面中间相中铝元素含量远大于铜/铝中间相中的 含量, 在 SEM 里显示的颜色更深。可以明显看到厚 度约为 $1.7 \mu \mathrm{m}$ 中间相层, 该层的主要扩散方向是 
$\mathrm{Fe}$ 扩散至 $\mathrm{Al}$ 中。焊接区域边缘处的中间相厚度明显 要小于中心处(图 13c)。对界面中心处(图 13b 中线条 以及点)进行 EDS 测试结果(图 13d)表明, 其中间相的 化学成分为 $47.9 \mathrm{Fe}-52.1 \mathrm{Al}(\%)$, 换算成原子占比为 $69.24 \mathrm{Fe}-30.76 \mathrm{Al}(\%)$ 。这表明的中间相成分最可能是 $\mathrm{Fe}_{2} \mathrm{Al}_{5}$ 。从接头断口表面获得的 XRD 光谱(图 14)也证 实了 $\mathrm{Fe}_{2} \mathrm{Al}_{5}$ 和 $\mathrm{FeAl}_{3}$ 相的存在, 但无法检测到 $\mathrm{FeAl}$ 相, 这是因为该中间相很薄, 所占的体积分数低。

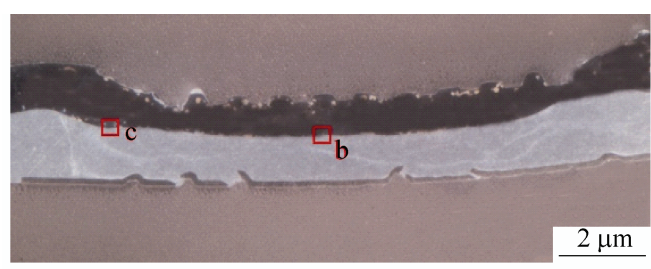

(a) 接头横截面形貌

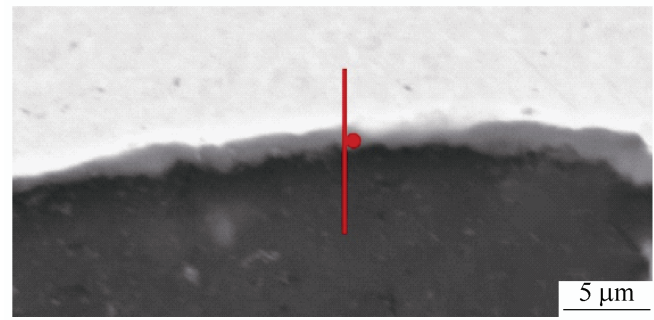

(b) 焊接区域中心处形貌

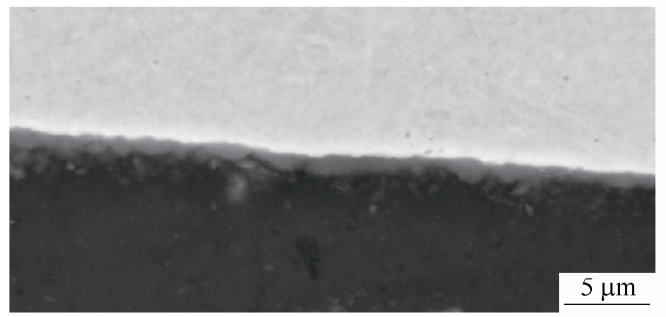

(c) 焊接边缘处形貌

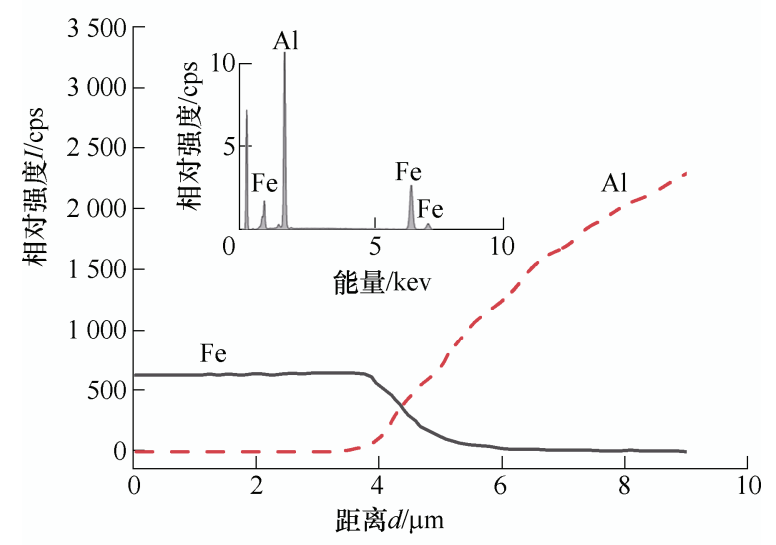

(d) EDS结果

图 13 接头 SEM 结果

图 15 为 SEM 观测的不同焊接时间的中间相形 貌。从 $15 \mathrm{a}$ 中可以看出, 即使是在很短的焊接时间, 界面也形成了较薄的不连续的 IMC 层。随着焊接时 间和温度的增加, 形成了连续的金属间层, 其厚度 迅速增加(图 $15 \mathrm{~b}$ 和 $15 \mathrm{c}$ ), 在焊接时间 $1.2 \mathrm{~s}$ 时生成
的中间相的厚度为 $2.2 \mu \mathrm{m}$ 。此外, 明显看到界面塑 性变形较大，有明显的机械互锁。

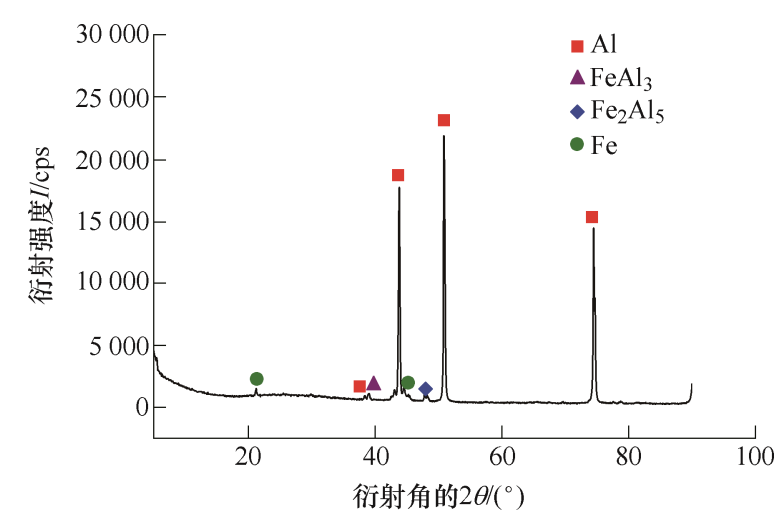

图 14 钢/铝接头断口 $\mathrm{Al}$ 侧 $\mathrm{XRD}$ 分析结果

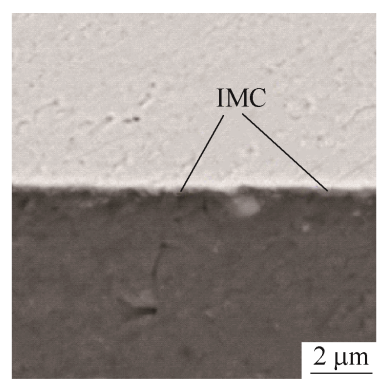

(a) $0.2 \mathrm{~s}$



(c) $0.8 \mathrm{~s}$

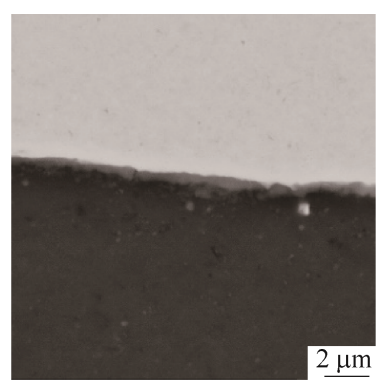

(b) $0.4 \mathrm{~s}$



(d) $1.2 \mathrm{~s}$
图 15 中间相形貌

图 16 为界面温度、中间相厚度随焊接时间的变 化关系。这可以用来推测超声作用下钢/铝界面的扩 散系数。根据菲克扩散定律, 中间相厚度 $l$ 与扩散 率 $D$ 及焊接时间 $t_{w}$ 关系为 ${ }^{[21]}$

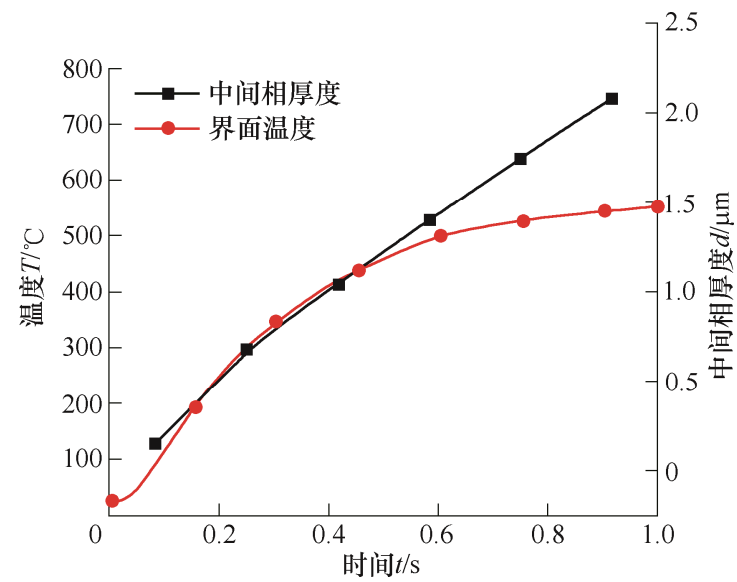

图 16 界面温度、中间相厚度随焊接时间的变化 


$$
l=\sqrt{D \times t_{\mathrm{w}}}
$$

根据公式推测在时间 $1 \mathrm{~s}$ 时候钢/铝扩散系数为 $2.89 \times 10^{-12} \mathrm{~m}^{2} / \mathrm{s}$, 这远高于激光焊接时的扩散率 $4.1 \times 10^{-13} \mathrm{~m}^{2} / \mathrm{s}^{[22]}$ 。这是因为相对于温度导致的晶界 扩散, 在超声振动下, 除了摩擦热产生的晶界扩散, 更多的是声场使材料空位浓度增加, 导致晶体内容 纳了大量的其他原子，产生了晶体内部扩散 ${ }^{[23]}$ 。

\subsection{2 断口形貌}

图 17 为焊接时间 $1 \mathrm{~s}$ 时钢/铝接头的断口形貌。 钢/铝接头断口中焊接区域面积约为 $9 \mathrm{~mm} \times 7 \mathrm{~mm}$ (图 17a), 明显大于焊头的面积 $7 \mathrm{~mm} \times 5 \mathrm{~mm}$ 。这是 因为钢/铝超声波焊接界面温度较高, 促进材料软 化, 在压力和剪切力的共同作用下, 铝和钢板均发 生塑性变形, 在高的压力作用下导致焊接区域的面 积比焊头端面大。在靠近焊接区域中心处(图 17a 中 的区域 b), 明显可见韧性为主并包含少数脆性的两 种不同的断裂模式。图 $17 \mathrm{c}$ 中看到大量不同尺寸的 韧窝区域。在靠近焊接区域边缘处(图 17a 中的区域 d), 明显可见脆性为主并包含少量韧窝, 这是因为 边缘处的中间相太薄不足以形成可靠连接(图 13c)。 总的来说, 钢/铝超声波焊接接头的断裂模式是韧 性-脆性复合断裂。

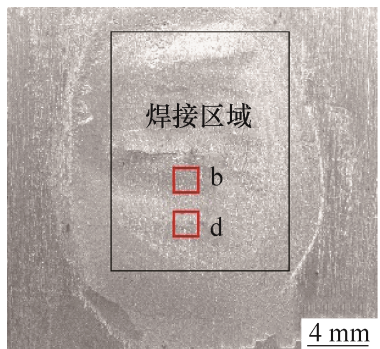

(a) 断口宏观形貌

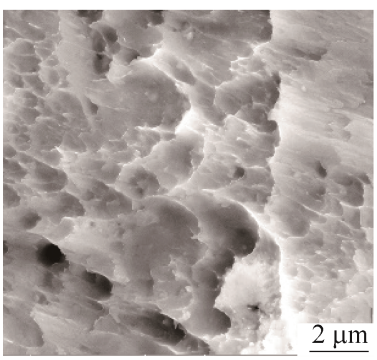

(c) 图17b放大图

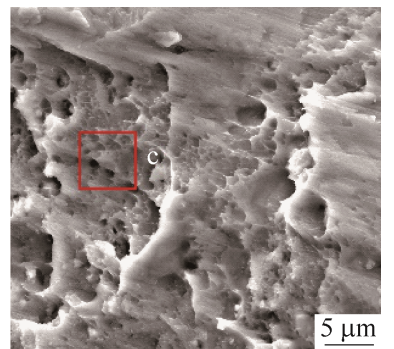

(b) 界面附近处形貌



(d) 界面边缘处形貌
图 17 断口形貌

\subsection{3 接头力学性能}

在焊接时间 $1 \mathrm{~s}$ 时获得的钢/铝超声波焊接接头 的最大拉伸剪切力约为 $3.95 \mathrm{kN}$ (图 18a), 将焊接区 域近似为图 $17 \mathrm{a}$ 中矩形面积, 所计算的焊接强度约 为 $63 \mathrm{MPa}$, 该强度明显高于钢/铝小功率超声波焊 接接头强度 ${ }^{[2]}$, 也高于 $\mathrm{ZHAO}$ 等 ${ }^{[24]}$ 获得的钢/铝大功
率超声波焊接接头强度, 同时比 HADDADI 等 ${ }^{[16]}$ 获得的在焊接时间 $1.5 \mathrm{~s}$ 时的大功率 $(2.5 \mathrm{~kW})$ 超声焊 钢/铝强度略高(约高 $0.5 \%$ ), 且焊接区域面积更大, 更利于在汽车轻量化的推广。图 18 为钢/铝接头剪 切力与焊接时间的关系。在较短时间的超声波焊接 里, 由于输入能量低, 界面存在的虚焊和刮擦区域 较多。随着焊接的进行, 界面连接点增多, 母材之 间的扩散会越充分, 强度也越高。但过长的焊接时 间导致中间相过厚以及焊接区域边缘处可能出现疲 劳裂纹。



(a) 接头拉伸曲线

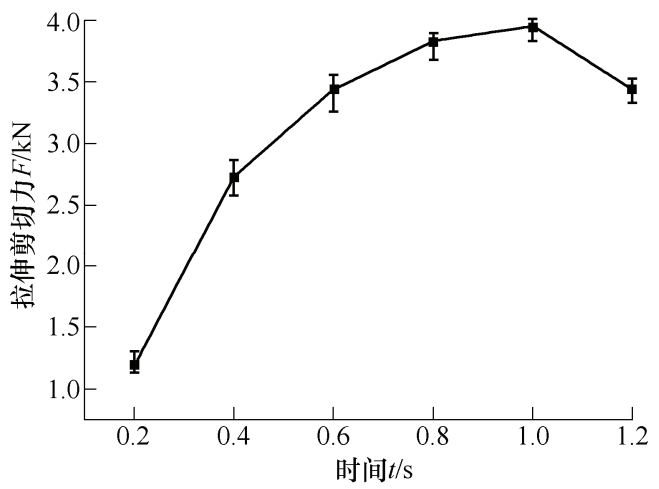

(b) 钢/铝接头拉伸强度随焊接时间变化

图 18 接头拉伸剪切力

异质接头超声波焊接强度取决于三个因素：界 面处的塑性应变、IMC 的成分和厚度和焊接线长度 (或焊接面积)。IMC 厚度对焊接接头抗拉强度的影 响如图 19 所示。很明显, 过小的 IMC 厚度导致界 面扩散不充分，连接质量差；但过大的 IMC 厚度同 样不利于焊接。对于钢/铝超声波焊接, 在 IMC 厚 度 $1.7 \mu \mathrm{m}$ 获得了较高质量的焊接接头, 这与 HADDADI 等 ${ }^{[16]}$ 报导的结论一致。大功率超声焊接 下获得的焊接面积较大(图 17a), 这也促进了连接。 此外, 工件的搭置顺序也会影响焊接质量。本研究 是将钢作为上工件, 这样大部分超声波振动能量传 递至钢中, 使钢软化程度高, 在高温和高声强密度 双重作用下界面变形更大。然而由于钢的刚度较高, 
过大的变形会破坏接头质量。但如果钢作为下工件, 其变软主要为温度作用, 导致内部塑性变形不足同 样难以形成高质量的焊接。因此, 对于钢/铝大功率 超声波焊接而言, 需要更优化的工艺参数来获得合 理的界面塑性变形。

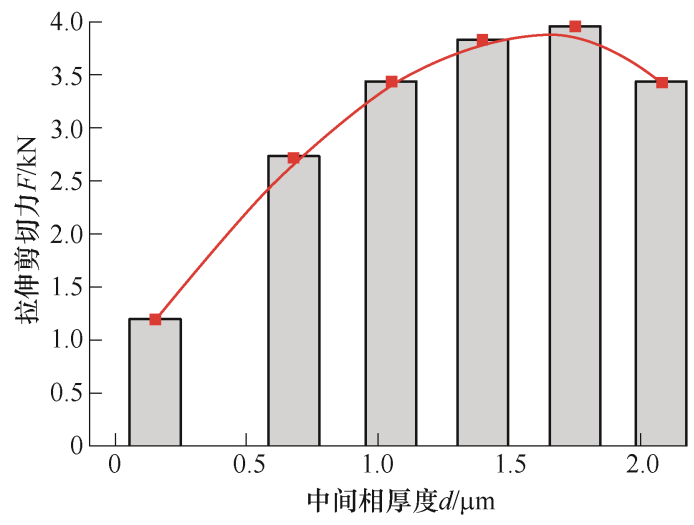

图 19 中间相厚度与拉伸剪切力关系

\section{3 结论}

(1) 在焊接初期, 界面处局部形成中间相, 随 着钢/铝超声波焊接过程的进行, 界面中间相逐渐增 厚。在焊接时间 $1 \mathrm{~s}$ 、压力 $1.98 \mathrm{kN}$ 、振幅 $27 \mu \mathrm{m}$ 条 件下, 焊接界面生成主要由 $\mathrm{Fe}_{2} \mathrm{Al}_{5}$ 组成、厚度为 $1.7 \mu \mathrm{m}$ 的中间相, 并获得钢/铝接头最高拉剪强度为 $3.95 \mathrm{kN}$ 。钢/铝界面变形较大, 存在明显的机械互锁 作用。在高温及高振幅作用下材料软化, 导致焊接 区域面积明显大于焊头端面。

(2) 在焊接时间 $0.54 \mathrm{~s}$ 、压力 $1.45 \mathrm{kN}$ 、振幅 $25 \mu \mathrm{m}$ 条件下, 界面生成主要成分为 $\mathrm{Al}_{2} \mathrm{Cu}$ 厚度为 $1.95 \mu \mathrm{m}$ 的中间相。获得的铜/铝接头的最高拉剪强 度分别为 $3.20 \mathrm{kN}$, 此时接头拉伸破坏为拉拔式破 坏。铜/铝焊接界面平直, 局部区域有漩浴状的塑性 变形。

(3) 模拟结果表明, 钢/铝焊接中有近 51\%的超 声功率转化为界面产热, 在时间 $1 \mathrm{~s}$ 时的温度升高 至铝合金熔点的约 $80 \%$; 超声作用下的钢/铝界面扩 散系数比温度作用下大一个数量级。

（4）铝合金异种金属超声波焊接质量与中间相厚 度有关，适当厚度的中间相促进了异质接头连接。铝 合金接头的拉伸断裂形式均为塑性-韧性混合断裂。

\section{参 考 文 献}

[1] 李永兵, 马运五, 楼铭, 等. 轻量化薄壁结构点连接技 术研究进展 [J]. 机械工程学报, 2020，56(6): 125-146. LI Yongbing, MA Yunwu, LOU Ming, et al. Advances in spot joining technologies of lightweight thin-walled structures[J]. Journal of Mechanical Engineering, 2020, 56(6): 125-146.

[2] WATANABE T, SAKUYAMA H, YANAGISAWA A. Ultrasonic welding between mild steel sheet and $\mathrm{Al}-\mathrm{Mg}$ alloy sheet[J]. Journal of Materials Processing Technology, 2009, 209(15-16): 5475-5480.

[3] NI Z L, YANG J J, GAO Z T, et al. Joint formation in ultrasonic spot welding of aluminum to copper and the effect of particle interlayer[J]. Journal of Manufacturing Processes, 2020, 50(2): 57-67.

[4] 王晓虹, 谷晓燕, 孙大千. 钢/铝异种金属激光焊接头 界面特性的研究 [J]. 机械工程学报, 2017, 53(4): 26-33. WANG Xiaohong, GU Xiaoyan, SUN Daqian. Research on interface characteristic of laser welding joints of steel/aluminum dissimilar materials[J]. Journal of Mechanical Engineering, 2017, 53(4): 26-33.

[5] LIANG Jianyong, LIANG Jun, FANG Guodong, et al. Evaluation on spot weld models in structural dynamic analysis of automotive body in white[J]. Chinese Journal of Mechanical Engineering，2011，24(1): 84-90.

[6] 武传松, 吕学奇, 宿浩, 等. 铝-镁异质合金搅拌摩擦 焊接成形的研究进展 [J]. 机械工程学报, 2020，56(6): 4-16.

WU Chuansong, LÜ Xueqi, SU Hao, et al. Research progress in dissimilar friction stir welding of aluminium/magnesium alloys[J]. Journal of Mechanical Engineering, 2020, 56(6): 4-16.

[7] 于明润, 赵洪运, 蒋智华, 等. 铝/黄铜异种金属搅拌 摩擦焊搭接接头显微组织与力学性能 [J]. 机械工程学 报，2019，55(6): 39-45.

YU Mingrun, ZHAO Hongyun, JIANG Zhihua, et al. Research on microstructure and mechanical properties of friction stir lap welded aluminum/brass dissimilar joint [J]. Journal of Mechanical Engineering, 2019， 55(6): 39-45.

[8] ZHAO Dewang, REN Daxin, ZHAO Kunming, et al. Ultrasonic welding of magnesium-titanium dissimilar metals : A study on thermo-mechanical analyses of welding process by experimentation and finite element method $[J]$. Chinese Journal of Mechanical Engineering, 2019，32(1): 1-11.

[9] BAKAVOS D, PRANGNELL P B. Mechanisms of joint and microstructure formation in high power ultrasonic spot welding 6111 aluminium automotive sheet[J]. Materials Science and Engineering: A，2010， 527(23): 6320-6334.

[10] SHAWN L S, HYUNG K T, JACK H S, et al. Analysis of weld formation in multilayer ultrasonic metal welding using high-speed images[J]. Journal of Manufacturing 
Science and Engineering, 2015, 137(3): 1-7.

[11] NONG L, SHAO C, KIM T H, et al. Improving process robustness in ultrasonic metal welding of lithium-ion batteries[J]. Journal of Manufacturing Systems, 2018, 48(7): $45-54$.

[12] FENG Mengnan, LUO Zhen. Interface morphology and microstructure of high-power ultrasonic spot welded $\mathrm{Mg} / \mathrm{Al}$ dissimilar joint[J]. Science and Technology of Welding and Joining, 2019, 24(1): 63-78.

[13] 谷晓燕, 刘婧, 刘东锋, 等. 焊接能量对 $\mathrm{Mg} / \mathrm{Al}$ 超声 波焊接接头微观组织与力学性能的影响 [J]. 机械工程 学报, 2019, 55(6): 23-31.

GU Xiaoyan, LIU Jing, LIU Dongfeng, et al. Effect of welding energy on microstructure and mechanical properties of $\mathrm{Mg} / \mathrm{Al}$ joints welded by ultrasonic spot welding[J]. Journal of Mechanical Engineering, 2019, 55(6): 23-31.

[14] LI Huan, CAO Biao, LIU Jian, et al. Modeling of high-power ultrasonic welding of $\mathrm{Cu} / \mathrm{Al}$ joint $[\mathrm{J}]$. The International Journal of Advanced Manufacturing Technology, 2018, 97(1-4): 833-844.

[15] 温昌金, 李玉龙, 赵诚. 铝合金/镀锌钢异种材料薄板 的超声波点焊 [J]. 焊接学报, 2015, 36(9): 39-42.

WEN Changin, LI Yulong, ZHAO Cheng. Ultrasonic spot welding of aluminum alloy and galvanized steel dissimilar metals sheets[J]. Transactions of the China Welding Institution, 2015, 36(9): 39-42.

[16] HADDADI F, ABU-FARHA F. Microstructural and mechanical performance of aluminium to steel high power ultrasonic spot welding[J]. Journal of Materials Processing Technology, 2015, 225(11): 262-274.

[17] JEDRASIAK P, SHERCLIFF H R. Finite element analysis of heat generation in dissimilar alloy ultrasonic welding[J]. Materials \& Design, 2018, 158(11): 184-197.

[18] KOMERLA K, NAUMOV A, MERTIN C, et al.
Investigation of microstructure and mechanical properties of friction stir welded AA6016-T4 and DC04 alloy joints[J]. The International Journal of Advanced Manufacturing Technology, 2018, 94(9-12): 4209-4219.

[19] ZHAO Yangyang, LI Dong, ZHANG Yansong. Effect of welding energy on interface zone of $\mathrm{Al}-\mathrm{Cu}$ ultrasonic welded joint $[\mathrm{J}]$. Science and Technology of Welding and Joining, 2013, 18(4): 354-360.

[20] YANG Jingwei, CAO Biao, LU Qinghua. The effect of welding energy on the microstructural and mechanical properties of ultrasonic-welded copper joints[J]. Materials, 2017, 10(2): 1-13.

[21] LI Huan, CAO Biao. Effects of welding pressure on high-power ultrasonic spot welding of $\mathrm{Cu} / \mathrm{Al}$ dissimilar metals[J]. Journal of Manufacturing Processes, 2019, 46(10): 194-203.

[22] FAN J, THOMY C, VOLLERTEN F. Effect of thermal cycle on the formation of intermetallic compounds in laser welding of aluminum-steel overlap joints[J]. Physics Procedia, 2011, 12(7): 134-141.

[23] GUNDUZ I, ANDO T, SHATTUCK E, et al. Enhanced diffusion and phase transformations during ultrasonic welding of zinc and aluminum[J]. Scripta Materialia, 2005, 52(9): 939-943.

[24] ZHAO Dewang, REN Daxin, ZHAO Kunming, et al. Effect of welding parameters on tensile strength of ultrasonic spot welded joints of aluminum to steel-By experimentation and artificial neural network[J]. Journal of Manufacturing Processes, 2017, 30(12): 63-74.

作者简介: 李欢, 男, 1983 年出生, 博士, 讲师。主要研究方向为异质 金属焊接工艺及过程信号挖掘。

E-mail: lihuan7@126.com

曹彪(通信作者), 男, 1963 年出生, 博士, 教授, 博士研究生导师。主 要研究方向为新型焊接技术及过程控制。

E-mail: mebcao@scut.edu.cn 